\title{
ON THE MAXIMAL OFFSPRING IN A CRITICAL BRANCHING PROCESS WITH INFINITE VARIANCE
}

\author{
JEAN BERTOIN, ${ }^{*}$ Université Pierre et Marie Curie
}

\begin{abstract}
We investigate the maximal number $M_{k}$ of offspring amongst all individuals in a critical Galton-Watson process started with $k$ ancestors. We show that when the reproduction law has a regularly varying tail with index $-\alpha$ for $1<\alpha<2$, then $k^{-1} M_{k}$ converges in distribution to a Frechet law with shape parameter 1 and scale parameter depending only on $\alpha$.
\end{abstract}

Keywords: Branching process; maximal offspring; Frechet distribution; stable Lévy process; extreme value theory

2010 Mathematics Subject Classification: Primary 60F05; 60J80

\section{Introduction and main results}

There exists in the literature a variety of results about extremes in branching processes; see the surveys by Yanev [11], [12] and the references therein. In particular, Rahinov and Yanev [9] have characterized the asymptotic behavior as $n \rightarrow \infty$ of the maximal offspring at the $n$th generation conditionally on the event that the extinction has not yet occurred. In a different direction, Pakes [8] considered the order statistic of scores in branching processes, and more precisely asymptotics of the largest score up to and including the $n$th generation, again conditionally on the event that extinction does not occur before the $n$th generation. In this vein, we also mention the recent contributions of Lebedev [6], [7] to the eternal branching process. The purpose of this work is to point at a simple result in this area which does not seem to have been observed before.

Let $F$ denote the distribution function of some probability measure on $\mathbb{Z}_{+}$, and write $\bar{F}=$ $1-F$ for its tail. We assume throughout this paper that

$$
\bar{F}(n)=n^{-\alpha} \ell(n),
$$

where $1<\alpha<2$ and $\ell$ is a slowly varying function. For each $k \geq 1$, we consider a BienayméGalton-Watson process $Z=\left(Z_{n}, n \geq 0\right)$ started from $Z_{0}=k$ ancestors and with reproduction law given by $F$. We are interested in the maximal offspring until extinction, viz.

$$
M_{k}=\max \left\{X_{i}: 1 \leq i \leq T_{k}\right\}
$$

where $T_{k}=\sum_{n=0}^{\infty} Z_{n}$ denotes the size of the total population and $X_{i}$ is the number of children of the $i$ th individual (the choice of the enumeration of individuals is obviously irrelevant).

Received 14 September 2010; revision received 15 December 2010.

* Postal address: Laboratoire de Probabilités et Modèles Aléatoires, Université Pierre et Marie Curie, 4 Place Jussieu, 75252 Paris Cedex 05, France. Email address: jean.bertoin@upmc.fr 


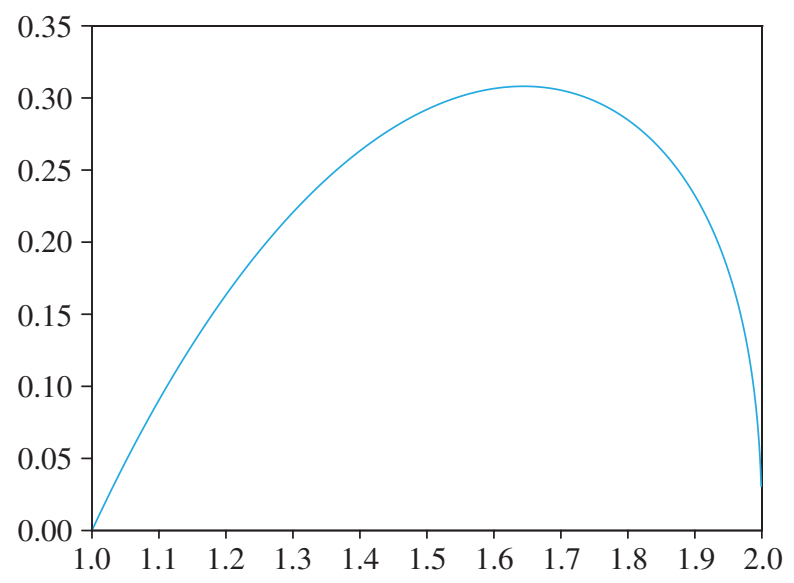

Figure 1: Graph of the map $\alpha \mapsto \beta$.

In the subcritical case $\sum_{i=0}^{\infty} \bar{F}(i)=m<1$, it is known from the law of large numbers that $T_{k} \sim k /(1-m)$ almost surely when $k \rightarrow \infty$, and it follows readily from extreme value theory that if we introduce a sequence $\left(n_{k}\right)_{k \in \mathbb{N}}$ such that $\bar{F}\left(n_{k}\right)=n_{k}^{-\alpha} \ell\left(n_{k}\right) \sim 1 / k$ then

$$
\lim _{k \rightarrow \infty} \mathrm{P}\left(M_{k} \leq x n_{k}\right)=\exp \left(-\frac{x^{-\alpha}}{1-m}\right), \quad x>0 .
$$

In the supercritical case $\sum_{i=0}^{\infty} \bar{F}(i)>1$, the probability of extinction tends to 0 as $k \rightarrow \infty$, and, therefore, so does the probability that $M_{k}<\infty$. We thus focus henceforth on the critical case

$$
\sum_{i=0}^{\infty} \bar{F}(i)=1
$$

and state our main result.

Theorem 1. Under assumptions (1) and (2), $k^{-1} M_{k}$ converges in distribution as $k \rightarrow \infty$ to a Frechet law with shape parameter 1 . More precisely, for every $x>0$, we have

$$
\lim _{k \rightarrow \infty} \mathrm{P}\left(M_{k} \leq k x\right)=\exp \left(-\frac{\beta}{x}\right),
$$

where the scale parameter $\beta>0$ is the unique solution to the equation

$$
\sum_{n=0}^{\infty} \frac{(-1)^{n} \beta^{n}}{(n-\alpha) n !}=0 .
$$

The graph of the map $\alpha \mapsto \beta$ is displayed in Figure 1 .

Of course, $M_{k}$ can be viewed as the maximum of $k$ independent and identically distributed (i.i.d.) copies of $M_{1}$, and we should expect a weak limit theorem for $M_{k}$ involving an extreme value distribution. Nonetheless, it is quite remarkable that the slowly varying function $\ell$ plays no role in this limit theorem, and that the index $\alpha$ appears only in the scale parameter $\beta$ of the limiting Frechet distribution, but not in the shape parameter. 
It is also interesting to note the following consequence of a classical result due to Gnedenko (see, for instance Proposition 1.11 of [10]) combined with Theorem 1.

Corollary 1. Under assumptions (1) and (2), the tail distribution of $M_{1}$ fulfills

$$
\mathrm{P}\left(M_{1}>x\right) \sim \frac{\beta}{x} \quad \text { as } x \rightarrow \infty,
$$

where $\beta>0$ is the unique solution to (3).

Note that $M_{1}$ thus has an infinite expectation even though the mean offspring of a typical individual is 1 .

By Gnedenko's theorem, the asymptotics stated in Theorem 1 and Corollary 1 are equivalent. Usually, it is more natural to establish first that the tail distribution of some random variable is regularly varying and to then deduce the limiting behavior of the maximum of i.i.d. copies. However, this is not the approach that we will use here. More precisely, hypothesis (1) is a necessary and sufficient condition for the distribution $F$ to belong to the domain of attraction of a spectrally positive stable law with index $\alpha$. Using the classical connection between downward skip-free random walks and branching processes, and standard arguments of weak convergence on the space of càdlàg paths, this enables us to reduce the proof of Theorem 1 to the determination of the distribution of the maximal size of jumps in a spectrally positive stable Lévy process up to its first hitting time of -1 . The latter is achieved by rather straightforward arguments of fluctuation theory for Lévy processes without negative jumps.

The proof of Theorem 1 is presented in the next section, and miscellaneous comments are made in Section 3.

\section{Proof of Theorem 1}

Introduce a sequence $X_{1}, X_{2}, \ldots$ of i.i.d. variables distributed according to $F$, and consider the downward skip-free random walk

$$
S_{n}=X_{1}+\cdots+X_{n}-n \text {. }
$$

It is a well-known fact observed by Harris (see Section 6 of [3]) that the Bienaymé-GaltonWatson branching process with reproduction law $F$ and started with $k$ ancestors can be constructed from the random walk stopped at its first hitting time of level $-k$,

$$
T_{k}=\min \left\{i: S_{i}=-k\right\} .
$$

In this setting, $T_{k}$ corresponds to the total size of the population generated by the branching process (so our notation is coherent) and the maximal offspring until extinction is closely related to the maximal step of the random walk until time $T_{k}$. More precisely, there is the identity

$$
M_{k}=\max \left\{X_{i}: 1 \leq i \leq T_{k}\right\} .
$$

Assumptions (1) and (2) ensure that the random walk $S$ belongs to the domain of attraction of a spectrally positive stable Lévy process with index $\alpha$, say $\xi=\left(\xi_{t}, t \geq 0\right)$. This means that there exists a sequence $\left(a_{k}\right)_{k \geq 1}$ of positive real numbers with $\lim _{k \rightarrow \infty} a_{k}=\infty$ such that

$$
\left(\frac{1}{k} S_{\left\lfloor a_{k} t\right\rfloor}, t \geq 0\right) \Rightarrow\left(\xi_{t}, t \geq 0\right),
$$

where ' $\Rightarrow$ ' denotes convergence in distribution as $k \rightarrow \infty$, in the sense of Skorokhod on the 
space of càdlàg paths. See, for instance, Theorem 16.14 of [4]. More precisely, $\xi$ is a process with independent and stationary increments, which fulfills the following scaling property:

$$
\text { for any } c>0,\left(c^{-1 / \alpha} \xi_{c t}, t \geq 0\right) \text { has the same law as } \xi \text {. }
$$

By standard arguments, convergence (4) can be extended to processes stopped at their first passage times, in the sense that if we introduce the first passage process

$$
\tau_{x}=\inf \left\{t:-\xi_{t}>x\right\}, \quad x \geq 0
$$

(so $\left(\tau_{x}\right)_{x \geq 0}$ is a stable subordinator with index $1 / \alpha$ ), then

$$
\left(\frac{1}{k} S_{\left\lfloor a_{k} t\right\rfloor}, t \leq \frac{T_{k}}{a_{k}}\right) \Rightarrow\left(\xi_{t}, t \leq \tau_{1}\right) .
$$

More precisely, although first passage times are discontinuous functionals for Skorokhod's topology, the function $x \mapsto \tau_{x}$ is continuous at each fixed $x \geq 0$ almost surely and in particular at $x=1$. This readily yields the sought extension. Because convergence in the sense of Skorokhod implies uniform convergence after suitable time changes, it follows that if we denote by $\Delta_{t}^{*}$ the largest jump made by $\xi$ on the time interval $[0, t]$, i.e.

$$
\Delta_{t}^{*}=\max \left\{\Delta_{s}=\xi_{s}-\xi_{s-}: 0 \leq s \leq t\right\},
$$

then

$$
\lim _{k \rightarrow \infty} \frac{1}{k} M_{k}=\Delta_{\tau_{1}}^{*} \quad \text { in distribution. }
$$

By Gnedenko's theorem, this entails that $\Delta_{\tau_{1}}^{*}$ has the Frechet distribution with shape parameter 1 , and we only need to determine its scale parameter. We state this as a lemma that may be of independent interest.

Lemma 1. Let $\xi$ be a spectrally positive stable Lévy process with index $\alpha \in(1,2)$. The distribution of the size of its largest jump until the hitting time of -1 is given by

$$
\mathrm{P}\left(\Delta_{\tau_{1}}^{*} \leq y\right)=\exp \left(-\frac{\beta}{y}\right), \quad y>0,
$$

where $\beta>0$ is the unique solution to (3).

Proof. Thanks to the scaling property, there is no loss of generality in assuming that the Lévy measure $\Pi$ of $\xi$ is simply

$$
\Pi(\mathrm{d} x)=x^{-\alpha-1} \mathrm{~d} x, \quad x>0 .
$$

Recall that the Laplace exponent $\Psi$ of $\xi$ is given by the Lévy-Khintchin formula

$$
\Psi(q)=\int_{0}^{\infty}\left(\mathrm{e}^{-q x}-1+q x\right) \Pi(\mathrm{d} x)=\frac{\Gamma(2-\alpha)}{\alpha(\alpha-1)} q^{\alpha}, \quad q \geq 0,
$$

and then

$$
\mathrm{E}\left(\exp \left(-q \xi_{t}\right)\right)=\exp (t \Psi(q))
$$


Fix $y>0$, and let $\delta_{y}=\inf \left\{t: \Delta_{t}>y\right\}$ denote the first instant when $\xi$ makes a jump of size greater than $y$. Also, introduce, for every $t \geq 0$,

$$
\xi_{t}^{y}=\xi_{t}-\sum_{0 \leq s \leq t} \Delta_{s} \mathbf{1}_{\left\{\Delta_{s}>y\right\}}
$$

i.e. $\xi^{y}=\left(\xi_{t}^{y}, t \geq 0\right)$ is the spectrally positive Lévy process obtained from $\xi$ by suppressing all the jumps of size greater than $y$. In particular, its Laplace exponent is given by

$$
\begin{aligned}
\Psi^{y}(q) & =\int_{0}^{y}\left(\mathrm{e}^{-q x}-1+q x\right) \Pi(\mathrm{d} x)+q \int_{y}^{\infty} x \Pi(\mathrm{d} x) \\
& =\int_{0}^{y}\left(\mathrm{e}^{-q x}-1+q x\right) x^{-1-\alpha} \mathrm{d} x+q \frac{y^{1-\alpha}}{\alpha-1} .
\end{aligned}
$$

Introduce now the first passage time of $\xi^{y}$ at -1 , i.e.

$$
\tau_{1}^{y}=\inf \left\{t: \xi_{t}^{y}=-1\right\}
$$

and note that the events $\left\{\Delta_{\tau_{1}}^{*} \leq y\right\}$ and $\left\{\tau_{1}^{y}<\delta_{y}\right\}$ coincide. Recall further from the Lévy-Itô decomposition of Lévy processes that $\xi^{y}$ and a fortiori $\tau_{1}^{y}$ are independent of $\delta_{y}$. Since the latter has the exponential distribution with parameter $\bar{\Pi}(y)=\Pi((y, \infty))=\alpha^{-1} y^{-\alpha}$, we deduce that

$$
\mathrm{P}\left(\Delta_{\tau_{1}}^{*} \leq y\right)=\mathrm{P}\left(\tau_{1}^{y}<\delta_{y}\right)=\mathrm{E}\left(\exp \left(-\alpha^{-1} y^{-\alpha} \tau_{1}^{y}\right)\right) .
$$

It is well known (see, for instance, Theorem VII.1 of [1] or Theorem 3.12 of [5]) that the right-hand side can be expressed as

$$
\mathrm{E}\left(\exp \left(-\alpha^{-1} y^{-\alpha} \tau_{1}^{y}\right)\right)=\exp (-b(y))
$$

where $b(y)>0$ is the unique solution to $\Psi^{y}(b(y))=\alpha^{-1} y^{-\alpha}$. Thus, we are led to solving the equation

$$
\int_{0}^{y}\left(\mathrm{e}^{-b(y) x}-1+b(y) x\right) x^{-1-\alpha} \mathrm{d} x+b(y) \frac{y^{1-\alpha}}{\alpha-1}=\alpha^{-1} y^{-\alpha} .
$$

The change of variable $x=y u$ yields

$$
\int_{0}^{1}\left(\mathrm{e}^{-b(y) y u}-1+b(y) y u\right) u^{-1-\alpha} \mathrm{d} u+\frac{b(y) y}{\alpha-1}=\frac{1}{\alpha},
$$

and setting $\beta=y b(y)$, we finally arrive at

$$
\int_{0}^{1}\left(\mathrm{e}^{-\beta u}-1+\beta u\right) u^{-1-\alpha} \mathrm{d} u+\frac{\beta}{\alpha-1}=\frac{1}{\alpha} .
$$

Using the series expansion of $\mathrm{e}^{-\beta u}$, we see that (3) and (5) are equivalent.

Finally, note that the function

$$
q \rightarrow \int_{0}^{1}\left(\mathrm{e}^{-q u}-1+q u\right) u^{-1-\alpha} \mathrm{d} u+\frac{q}{\alpha-1}
$$

is convex and strictly increasing on $[0, \infty)$, and, thus, (5) has a unique solution. 


\section{Some comments}

1. Amateurs of special functions will recognize that (3) can be re-expressed in terms of the incomplete gamma function. Specifically, recall that, for $\operatorname{Re}(a)>0$,

$$
\gamma(a, x)=\int_{0}^{x} \mathrm{e}^{-t} t^{a-1} \mathrm{~d} t=\sum_{n=0}^{\infty} \frac{(-1)^{n} x^{a+n}}{(a+n) n !}
$$

is the incomplete gamma function; see Equation 8.354.1 of [2]. The modified function

$$
\gamma^{*}(a, x)=\frac{x^{-a}}{\Gamma(a)} \gamma(a, x)
$$

is analytic with respect to $a$ and $x$ (cf. Equation 8.353.1 of [2]), and, therefore, the solution $\beta$ to (3) is the unique positive root to the equation $\gamma^{*}(-\alpha, \beta)=0$.

2. In connection with Lemma 1 , we recall that the distribution of the maximal jump size up to time $t, \Delta_{t}^{*}$, is given by

$$
\mathrm{P}\left(\Delta_{t}^{*} \leq x\right)=\exp (-t \bar{\Pi}(x))=\exp \left(-t c x^{-\alpha}\right)
$$

where $\bar{\Pi}$ stands for the tail of the Lévy measure and $c>0$ is some constant. Let us assume for simplicity that $\xi$ is normalized as in the proof of Lemma 1 , so that $c=1 / \alpha$. In this situation, the Laplace transform of $\tau_{1}$ is

$$
\mathrm{E}\left(\exp \left(-q \tau_{1}\right)\right)=\left(\frac{\alpha(\alpha-1)}{\Gamma(2-\alpha)} q\right)^{1 / \alpha}
$$

and we deduce that if $T$ is a random time distributed as $\tau_{1}$ and independent of $\xi$, then

$$
\mathrm{P}\left(\Delta_{T}^{*} \leq x\right)=\mathrm{E}\left(\exp \left(-\frac{1}{\alpha x^{\alpha}} \tau_{1}\right)\right)=\exp \left(-\left(\frac{\alpha-1}{\Gamma(2-\alpha)}\right)^{1 / \alpha} x^{-1}\right) .
$$

We stress that, thanks to the scaling property, the distribution of $\Delta_{T}^{*}$ does not depend on the normalization that has been chosen for $\xi$. We see that $\Delta_{T}^{*}$ has again the Frechet law with shape parameter 1 , but the scale parameter differs from the solution to (3).

\section{Acknowledgements}

I would like to thank George Yanev for pointing at interesting references. This work has been supported by ANR-08-BLAN-0220-01.

\section{References}

[1] Bertoin, J. (1996). Lévy Processes. Cambridge University Press.

[2] Gradshteyn, I. S. and Ryzhik, I. M. (2007). Table of Integrals, Series, and Products, 7th edn. Elsevier/Academic, Amsterdam.

[3] Harris, T. E. (1952). First passage and recurrence distributions. Trans. Amer. Math. Soc. 73, 471-486.

[4] Kallenberg, O. (2002). Foundations of Modern Probability, 2nd edn. Springer, New York.

[5] Kyprianou, A. E. (2006). Introductory Lectures on Fluctuations of Lévy Processes with Applications. Springer, Berlin.

[6] Lebedev, A. V. (2008). Maxima of random properties of particles in supercritical branching processes. Moscow Univ. Math. Bull. 63, 175-178. 
[7] LebedeV, A. V. (2008). Maxima of random particles scores in Markov branching process with continuous time. Extremes 11, 203-216.

[8] PaKes, A. G. (1998). Extreme order statistics on Galton-Watson trees. Metrika 47, 95-117.

[9] Rahimov, I. and Yanev, G. P. (1999). On maximum family size in branching processes. J. Appl. Prob. 36, 632-643.

[10] ReSnick, S. I. (1987). Extreme Values, Regular Variation, and Point Processes. Springer, New York.

[11] Yanev, G. P. (2007). Revisiting offspring maxima in branching processes. Pliska Stud. Math. Bulgar. 18, 401426.

[12] Yanev, G. P. (2008). A review of offspring extremes in branching processes. In Records and Branching Processes, eds M. Ahsanullah and G. P. Yanev, Nova Science, pp. 127-145. 